\title{
Non-invasive assessment of the donor corneal endothelium using ocular redox fluorometry
}

\author{
J Shimazaki, R A Laing, K Tsubota, K R Kenyon
}

\begin{abstract}
Aims-To investigate the usefulness of ocular redox fluorometry for evaluating donor corneal endothelial viability.

Methods-Corneas from 42 recipients of penetrating keratoplasty and four donor corneas were examined by ocular redox fluorometry. Autofluorescence from reduced pyridine nucleotides (PN) and oxidised flavoproteins (Fp) of the human corneal endothelium were measured noninvasively, and the PN/Fp ratio was used as a tissue metabolic indicator. Specular microscopy and electron microscopy were also performed.
\end{abstract}

Results-Both the quality of specular microscopic image and the PN/Fp ratio were significantly correlated with the degree of corneal endothelial damage determined by histological examination. Corneas with poor specular microscopic image showed significantly decreased PN/Fp ratio compared with corneas with good or fair specular images $(p=0 \cdot 041$ and 0.027 , respectively). The $P N / F p$ ratio increased in corneas with mildly damaged endothelium but decreased in corneas with severely damaged endothelium determined by histological examination. Evaluation of corneal endothelium by combination of specular microscopy and ocular redox fluorometry showed excellent association with that of histopathological examination $(\mathbf{p}<0.0001)$.

Conclusion-Ocular redox fluorometry is useful for assessing donor corneal endothelial viability. Combination of ocular redox fluorometry and specular microscopy may increase the ability of donor cornea selection.

(Brf Ophthalmol 1996; 80: 69-73)

Proper evaluation of donor corneas is the key to success in corneal transplantation. To date, slitlamp examination is the objective method being routinely used in eye banks, and now specular microscopy has been introduced in many eye banks. Although it has been shown that morphometric alterations of corneal endothelium correlate with donor cornea viability, ${ }^{1}$ a more sensitive and accurate measurement technique than specular microscopy is necessary for reliable assessment of the corneal condition. Nuclear magnetic resonance (NMR) is a noninvasive method for the measurement of metabolism, and it has been shown to be capable of assessing metabolic changes in donor corneas. ${ }^{2}$ However, at present resolutions the method cannot distinguish metabolic changes in endothelium from those in other layers. In addition, the complexity and high cost prevent the method from being introduced in most eye banks.

Ocular redox fluorometry is an alternative non-invasive method for measuring tissue metabolic changes. ${ }^{3-8}$ It measures autofluorescence from reduced pyridine nucleotides $(\mathrm{PN})$ and oxidised flavoproteins (Fp). These signals have been demonstrated to be valuable for the detection of metabolic changes in cornea and lens under a variety of conditions. ${ }^{3-8}$ The $\mathrm{PN} / \mathrm{Fp}$ ratio has been shown to be an especially sensitive indicator of tissue metabolic changes, since the ratio is less susceptible to tissue movements during the measurement than is each signal separately. ${ }^{9}$ The ratio was also shown to change in preserved rabbit corneal endothelium as a function of storage time. ${ }^{5} \mathrm{We}$ thus expect the method to be valuable for donor cornea selection.

In the present study, we investigated whether or not the method was valuable for detecting metabolic changes in the human corneal endothelium in eye banks. Corneas from recipients of corneal transplantation were used. Association between ocular redox fluorometry or specular microscopy and histopathology was studied.

\section{Materials and methods}

PATIENT PROFILE

Forty two corneas from recipients of penetrating keratoplasty were obtained at the time of surgery. Mean age of the recipients was $66 \cdot 1$ (SD 17.4) years, with the male to female ratio of 1:1. The leading cause of keratoplasty was bullous keratopathy $(n=23)$, followed by Fuchs' dystrophy $(n=8)$, keratoconus $(n=4)$, and regraft $(n=4)$. After the corneal buttons were removed, they were stored in the refrigerator overnight with McCarey-Kaufman (MK) medium, and examinations were performed thereafter.

Four corneas from four donors, which were rejected for keratoplasty because they failed to pass virus serology, were also studied. Three of these donors were male and one was female, with the mean age of 41.8 years.

\section{SPECULAR MICROSCOPY}

Corneal endothelium of the 36 corneas (32 recipient and four donor corneas) were evaluated by a single investigator (JS) using specular microscopy (Bio-Optics 2100C, Arlington, MA, USA) and the image was recorded with a video recorder. The specular microscopic 
Table 1 Semiquantitative assessment of corneal endothelium

\begin{tabular}{lllll}
\hline Endothelial damage & $\begin{array}{l}\text { Enlargement } \\
\text { of the cell }\end{array}$ & $\begin{array}{l}\text { Cell surface } \\
\text { change }\end{array}$ & $\begin{array}{l}\text { Changes in } \\
\text { cell junction }\end{array}$ & $\begin{array}{l}\text { Descemet's } \\
\text { membrane exposure }\end{array}$ \\
\hline Normal & No & No & No & No \\
Mildly damaged & Mild & Mild & Mild & No \\
Moderately damaged & Moderate & Moderate & Moderate & Mild \\
Severely damaged & Severe & Severe & Severe & Moderate to severe \\
\hline
\end{tabular}

*Including changes of microvilli and microplicae.

tIncluding loss of cellular junction, development of cytoplasmic extensions.

image was assessed semiquantitatively as follows: good - the cell borders were easily distinguished in most areas; fair - the cell borders were distinguished in some areas; poor the cell borders were not detected in most areas.

\section{OCULAR REDOX FLUOROMETRY}

After the specular microscopic examination, the cornea immersed in MK medium was placed under an ocular redox fluorometer with endothelial side up. The cornea was kept at room temperature during the measurement to produce a better appearance of the endothelium, as is performed in eye banks. Autofluorescence from PN (excitation $366 \mathrm{~nm}$ and emission $438 \mathrm{~nm}$ in wavelength) and $\mathrm{Fp}$ (excitation $440 \mathrm{~nm}$ and emission $540 \mathrm{~nm}$ in wavelength) was measured as described previously. ${ }^{3-8}$

The PN and Fp signals were measured in two ways. In the first 30 corneas, reflected light $(540 \mathrm{~nm}$ band path filters were used for both excitation and emission) was focused on the endothelium and the signals were measured at the focal plane. Secondly, the microscope stage on which the cornea was placed was moved towards the microscope objective lens every $20 \mu \mathrm{m}$ from about $100 \mu \mathrm{m}$ above to $200 \mu \mathrm{m}$ below the endothelium. Reflected light was measured simultaneously and it was used for

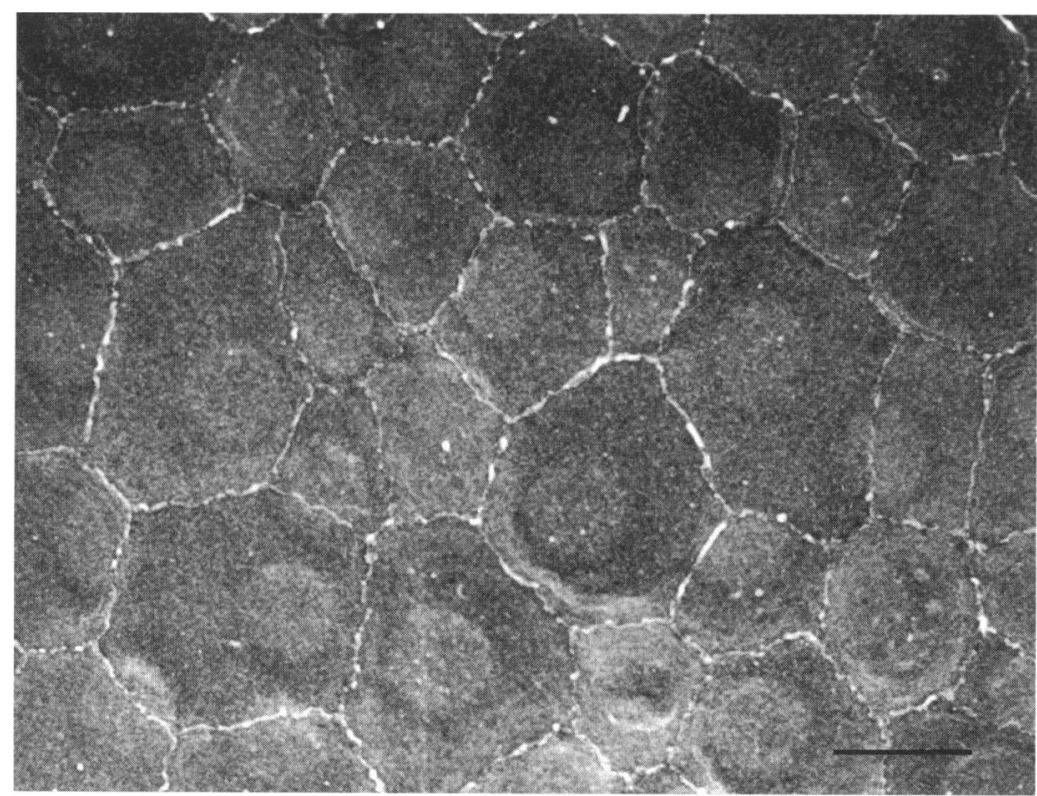

Fig $1 A$

Figure 1 Scanning electron micrographs of the diseased human corneal endothelium (bars $=10 \mu \mathrm{m}$ ). (A) Normal corneal endothelium. (B) Mildly damaged corneal endothelium. Note that endothelium is enlarged. (C) Moderately damaged endothelium. Each cell is further enlarged and exposed Descemet's membrane is seen. (D) Severely damaged endothelium. A large area of exposed Descemet's membrane is seen. the determination of the endothelial surface position. This latter method has advantages for the elimination of effects of tissue movement during measurement. ${ }^{6-8}$ However, the signals obtained by both methods demonstrated good agreement and so the results were combined. Each measurement was repeated three times and the average of these results was used. The $\mathrm{PN} / \mathrm{Fp}$ ratio, which has been shown to be a sensitive indicator of the tissue metabolic states, ${ }^{9}$ was calculated by an attached computer.

\section{HISTOPATHOLOGICAL EVALUATION}

After the fluorometric measurement, 32 of the 42 recipient corneas and four donor corneas were subjected to histological examination. These corneas were fixed by a half strength Karnovski's fixative, and processed for scanning (SEM: AMR 1000) and transmission (TEM: Philips 410 TER) electron microscopy.

Based on the SEM and TEM of the endothelium, the corneas were divided into four groups according to the criteria shown in Table 1 ; they were normal, mildly damaged, moderately damaged, and severely damaged. Representative photographs of each group are shown in Figures 1 and 2. This semiquantitative evaluation was performed by three independent examiners, who were not informed of the patients' data.

For the most part, all of the fluorometric and morphological assessments were made at the centre of the cornea. If the cell image could only be obtained away from the centre, specular microscopy and ocular redox fluorometry were performed in that region. In these instances, the location was recorded and histopathological evaluation was made for the same region.

Statistical calculation was performed using one way ANOVA and the non-paired $t$ test where indicated. A p value of less than 0.05 was regarded as significant.

\section{Results}

SPECULAR MICROSCOPY AND HISTOLOGICAL EXAMINATION

The histopathological evaluation of the three observers showed 0 or 1 grade differences in all corneas. Corneas which had different scores were considered to belong to the group that two of the observers had scored. Table 2 summarises the results of specular microscopy in each disease. Most of the corneas with bullous keratoplasty and from regrafts showed fair or poor specular images, and all of the donor corneas and keratoconus showed good specular image.

The quality of the specular microscopic image was significantly correlated with the results of the histological examination (Table $3, \chi^{2}$ test, $\left.\mathrm{p}<0 \cdot 0001\right)$. All corneas with histologically normal endothelium revealed a good specular image, and all corneas with severely damaged endothelium revealed fair or poor specular images. 


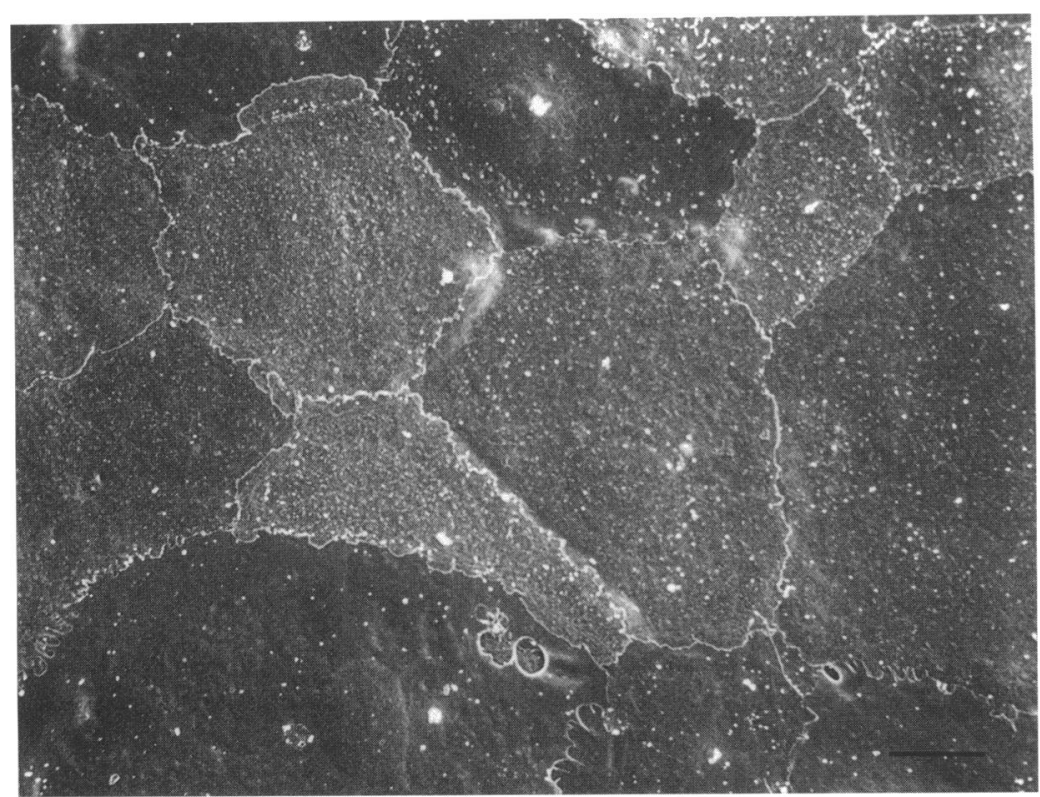

Fig $1 B$

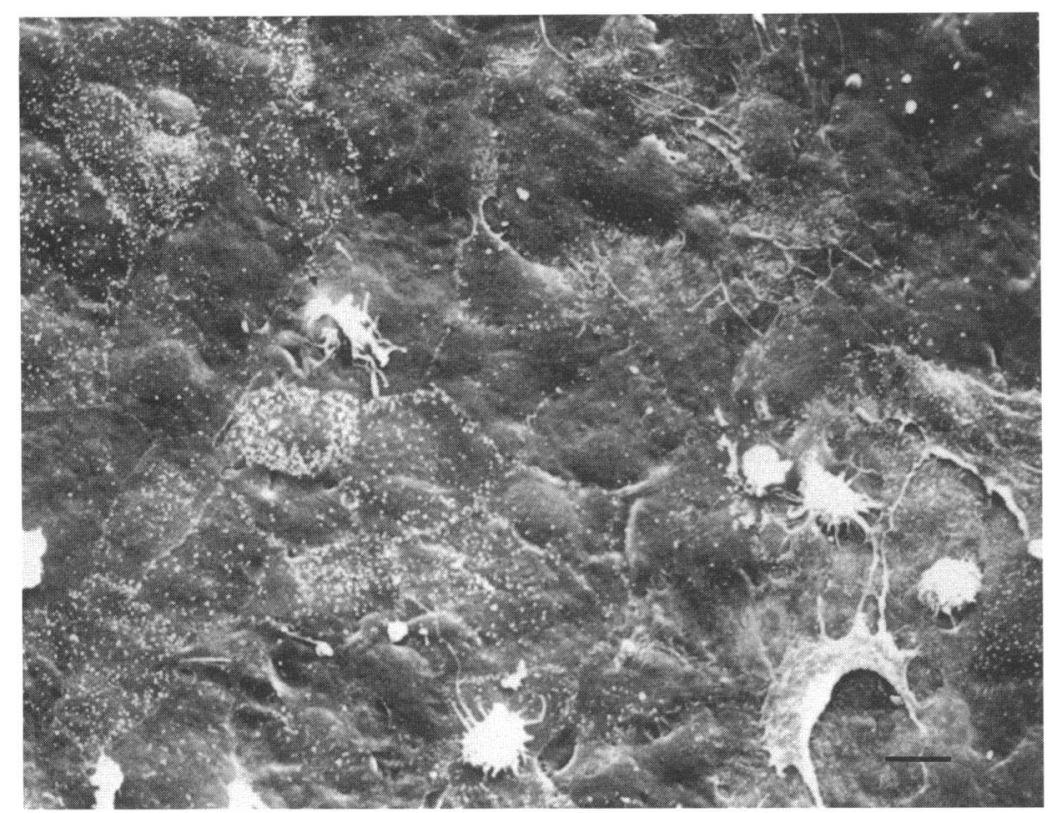

Fig $1 C$

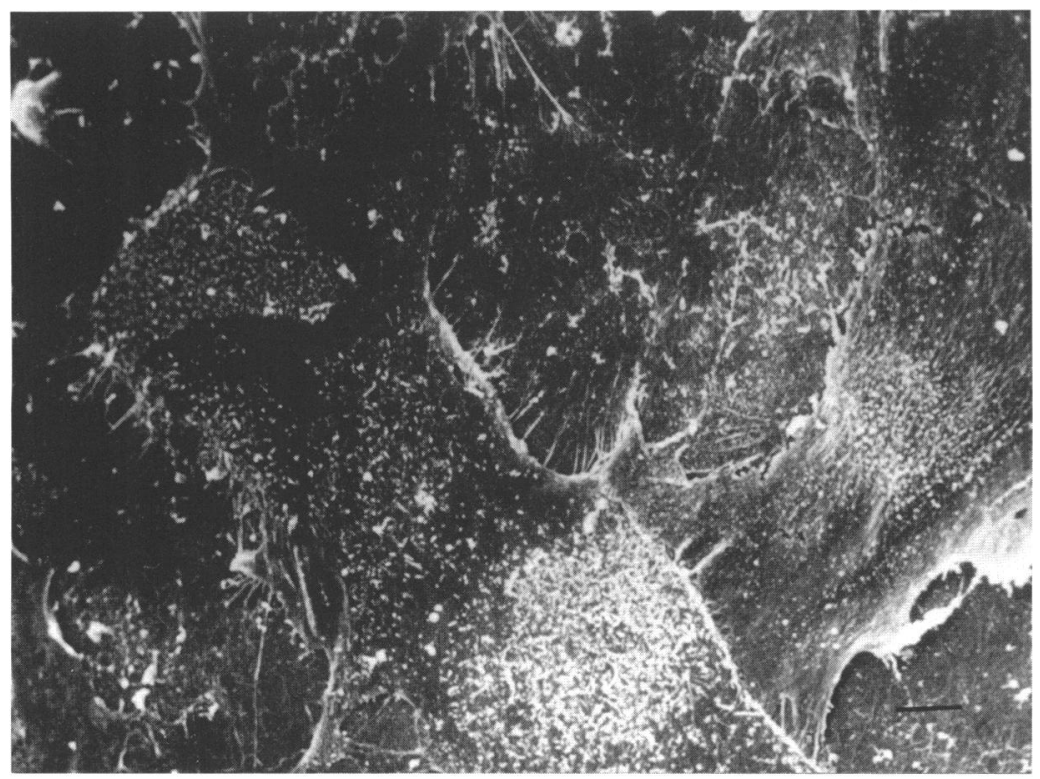

Fig $1 D$
SPECULAR MICROSCOPY AND OCULAR REDOX FLUOROMETRY

The PN signal and the PN/Fp ratio showed significant differences among groups of different specular microscopic images ( $p=0.046$ and $p=0.042$, respectively). The Fp signal did not differ significantly among these groups. The $\mathrm{PN} / \mathrm{Fp}$ ratio of the corneas with poor specular image was significantly increased in comparison with corneas with good or fair specular images $(p=0.041$ and $p=0.027$, respectively).

\section{HISTOPATHOLOGY AND OCULAR REDOX}

FLUOROMETRY

Seven, six, 14, and nine corneas, respectively, were categorised as having normal, mildly damaged, moderately damaged, and severely damaged corneal endothelium by histological examination (Table 4). As with specular microscopy, corneas with bullous keratopathy and from regrafts tended to have more damaged endothelium compared with keratoconus and donor corneas.

The PN signal and the PN/Fp ratio showed significant differences among these groups $(p=0.044$ and 0.001 , respectively). Although the Fp signal tended to increase as the severity of the endothelial damage advanced, the difference was not statistically significant $(p=0.061)$. In corneas with mildly damaged endothelium, the PN signal significantly increased $(p=0.0087)$. This change caused increases in the $\mathrm{PN} / \mathrm{Fp}$ ratio over those of the normal, moderately damaged, and severely damaged groups (Fig 3, p $=0.078,0.0058$, and 0.0007 , respectively). Both the PN and Fp signals increased in corneas with moderately damaged endothelium; however, both of them overlapped considerably with other groups. The PN/Fp ratio in this group showed much less overlap with the other groups than did the PN or Fp signals separately, and the ratio in this group showed significant differences compared with that in the mildly damaged and severely damaged groups. The PN/Fp ratio dramatically decreased in the severely damaged group. The PN signal was significantly decreased and the Fp signal was increased in this group compared with those in the normal group. Standard deviations of these signals and the PN/Fp ratio were small.

INTEGRATED EVALUATION OF CORNEAL ENDOTHELIUM BY SPECULAR MICROSCOPY AND OCULAR REDOX FLUOROMETRY

Based on the results of specular microscopy and ocular redox fluorometry, we attempted to evaluate corneal endothelial viability by the combination of these two methods. We use mean (1 SD) $(1 \cdot 29-2 \cdot 65)$ as a normal PN/Fp value. As shown in Table 5, corneas with high (more than 2.66) and low (less than 1.28) PN/Fp values were considered to be in the mildly and severely damaged endothelium, respectively. Corneas with a normal PN/Fp value were further divided into subgroups based on their specular microscopic images.

As a result six, seven, 10 , and 12 corneas, 


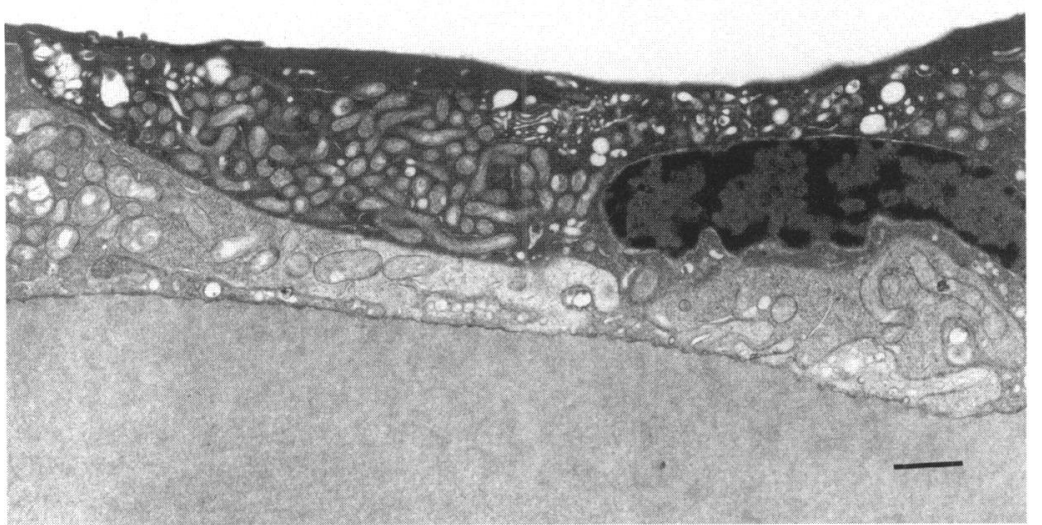

Fig $2 A$
Table 2 Specular microscopic image in various diseased corneas

\begin{tabular}{llll}
\hline \multirow{4}{*}{ Specular image } \\
\cline { 2 - 4 } Diseases & Good $(n=13)$ & Fair $(n=17)$ & Poor $(n=6)$ \\
\hline Bullous keratopathy & 3 & 9 & 4 \\
Fuchs' & 2 & 5 & 0 \\
Keratoconus & 3 & 0 & 0 \\
Regrafts & 0 & 2 & 2 \\
Scar & 1 & 1 & 0 \\
None (donor & & & \\
$\quad$ corneas) & 4 & 0 & 0 \\
\hline
\end{tabular}

respectively, were categorised into normal, mildly damaged, moderately damaged, and severely damaged groups. The result showed a significant correlation with that from histological examination (Table $6, \chi^{2}$ test, $\mathrm{p}<0.0001$ ). Twenty six out of 35 corneas $(74 \cdot 3 \%)$ revealed the same results from the two methods. Only two corneas revealed discordant results for more than two grades. (One normal cornea was judged as having severely damaged endothelium, and other eye with moderately damaged endothelium was judged as having normal endothelium.)

\section{Discussion}

In the present study, it was shown that changes in the ocular redox fluorometric signals were associated with histological findings of the recipient corneal endothelium. In corneas with mildly damaged endothelium determined by histology, the PN/Fp ratio significantly increased. TEM of corneas in this group showed thickened endothelium that contained abundant cellular organelles such as mitochondria. These findings suggest that the endothelial cells are under increased metabolic work load. ${ }^{10} \mathrm{~A}$ similar finding has been made in wound healing, when the PN/Fp ratio was shown to be increased. ${ }^{11}$

In corneas with moderately damaged endothelium determined by SEM, a variety of pathological changes were observed. It was not uncommon for some of the endothelial cells to be thickened and contain many mitochondria, while other cells in the same cornea were flattened with scattered intracellular organelles. Such regional differences in metabolic activity have been studied using histochemical techniques. ${ }^{12}$ A large standard deviation of the $\mathrm{PN} / \mathrm{Fp}$ ratio observed in the present study is likely to reflect such heterogeneity of cellular activity. Although efforts were made to perform all three measurements in the same region, the small measurement regions, about $150 \times 10 \mu \mathrm{m}$ with ocular redox fluorometry

Table 3 Association of specular microscopy and histopathological evaluation

\begin{tabular}{lllll}
\hline \multicolumn{5}{c}{ Histopathological evaluation } \\
\cline { 2 - 5 } $\begin{array}{l}\text { Specular } \\
\text { image }\end{array}$ & Normal & $\begin{array}{l}\text { Mildly } \\
\text { damaged }\end{array}$ & $\begin{array}{l}\text { Moderately } \\
\text { damaged }^{\star}\end{array}$ & $\begin{array}{l}\text { Severely } \\
\text { damaged }\end{array}$ \\
\hline Good & 7 & 3 & 2 & 0 \\
Fair & 0 & 3 & 10 & 4 \\
Poor & 0 & 0 & 1 & 5
\end{tabular}

*Specular microscopic examination was not available in one cornea of this group.
Figure 2 Transmission electron micrographs of the diseased human corneal endothelium. (A) Mildly damaged corneal endothelium (bar=1 $\mu \mathrm{m}$ ). Note that there are abundant intracellular organelles. (B) Moderately damaged endothelium. Endothelium is flattened and there are few intracellular organelles (bar $=1 \mu \mathrm{m}$ ). (C) Severely damaged endothelium. There are many cytoplasmic processes (bar $=10 \mu \mathrm{m}$ ). 
Table 4 Results of histopathological examination

\begin{tabular}{lllll}
\hline & \multicolumn{2}{l}{ State of endothelium } & \\
\cline { 2 - 5 } Diseases & $(n=7)$ & $\begin{array}{l}\text { Mildly } \\
\text { Normal } \\
(n=6)\end{array}$ & $\begin{array}{l}\text { Moderately } \\
\text { damaged } \\
(n=14)\end{array}$ & $\begin{array}{l}\text { Severely } \\
\text { damaged } \\
(n=9)\end{array}$ \\
\hline Bullous keratopathy & 0 & 1 & 8 & 7 \\
Fuchs' & 0 & 2 & 4 & 0 \\
Keratoconus & 3 & 0 & 1 & 2 \\
Regrafts & 0 & 1 & 1 & 0 \\
Scar & 0 & 2 & 0 & 0 \\
None (donor corneas) & 4 & 0 & 0 & 2 \\
\hline
\end{tabular}

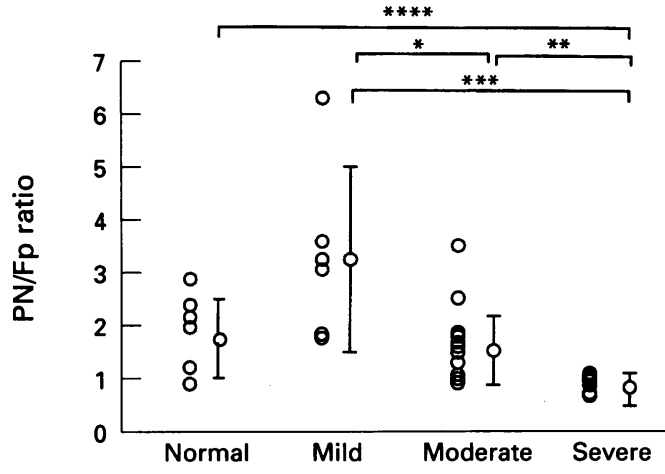

Figure 3 The PN/Fp ratio in corneas with different degrees of endothelial damage determined by histological examination. ${ }^{\star} p<0.0058,{ }^{\star \star} p=0.0020,{ }^{\star \star \star} p=0.0007$ $\star \star \star \star p=0.0005$.

and about $100 \times 80 \mu \mathrm{m}$ with histopathology, made the influence of heterogeneity inevitable.

In the severely damaged endothelium examined by SEM, the PN/Fp ratio decreased. The endothelial surface of these corneas was often denuded, and Descemet's membrane was exposed. Therefore, the fluorometry probably measured the signals from exposed Descemet's membrane. Low intensities of the PN and Fp signals observed in such corneas are similar to the signals in the corneal stroma. Additionally, extremely flattened endothelium may only weakly fluoresce as it contains few mitochondria, a main source of the fluorometric signals.

Table 5 Integrated assessment of the corneal endothelium by using both specular microscopy and ocular redox fluorometry

\begin{tabular}{|c|c|c|c|}
\hline \multirow[b]{2}{*}{$P N / F p$ value } & \multicolumn{3}{|l|}{ Specular image } \\
\hline & Good & Fair & Poor \\
\hline $\begin{array}{l}\text { Low }(<1 \cdot 28) \\
\text { Normal }(1 \cdot 29-2 \cdot 65) \\
\text { High }(>2 \cdot 66)\end{array}$ & $\begin{array}{l}\text { Severely damaged } \\
\text { Normal } \\
\text { Mildly damaged }\end{array}$ & $\begin{array}{l}\text { Severely damaged } \\
\text { Moderately damaged } \\
\text { Mildly damaged }\end{array}$ & $\begin{array}{l}\text { Severely damaged } \\
\text { Moderately damaged } \\
\text { Mildly damaged }\end{array}$ \\
\hline
\end{tabular}

Table 6 Association of the integrated assessment of the corneal endothelium and histological examination

\begin{tabular}{lllll}
\hline & \multicolumn{4}{l}{ Histological examination } \\
\cline { 2 - 5 } Integrated assessment & Normal & Mildly damaged & Moderately damaged & Severely damaged \\
\hline Normal & 4 & 1 & 1 & 0 \\
Mildly damaged & 2 & 4 & 1 & 0 \\
Moderately damaged & 0 & 1 & 9 & 0 \\
Severely damaged & 1 & 0 & 2 & 9 \\
\hline
\end{tabular}

For application of ocular redox fluorometry in an eye bank system, integrated assessment with specular microscopy is valuable (Table 6). Although specular microscopy showed a significant correlation with histological examination, five out of nine corneas with good specular image had mildly to moderately damaged endothelium determined by histological examination (Table 3). On the other hand, ocular redox fluorometry does not distinguish readily between normal and severely damaged endothelium. However, as shown in Table 6 , both together can distinguish between good, mildly damaged, and severely damaged endothelia. Ocular redox fluorometry probably would be a valuable tool for distinguishing between normal and mildly damaged endothelium in eye banks. Since most of the corneal endothelium examined in the present study was damaged, we could not study the relation between the endothelial density and redox fluorometry. Further studies should be performed using corneas with normal and mildly damaged endothelium.

In conclusion, ocular redox fluorometry was capable of detecting changes in the donor corneal endothelium. The $\mathrm{PN} / \mathrm{Fp}$ ratio measured by the method probably reflects metabolic differences in the endothelium. Because of its non-invasive nature, the method, especially when combined with specular microscopy, seems to be useful for donor cornea assessment.

The authors thank P John Anderson, $\mathrm{PhD}$ and Setsuko Oak for reviewing the manuscript.

1 Rao GN, Shaw EL, Arthur EJ, Aquavella JV. Endothelial cell morphology and corneal deturgescence. $A n n$ cell morphology and corneal

2 Greiner JV, Lass JH, Glonek T. Ex vivo metabolic analysis of eye bank corneas using phosphorus nuclear magnetic resonance. Arch Ophthalmol 1984; 102: 1171-3.

3 Laing RA, Fischbarg J, Chance B. Noninvasive measurements of pyridine nucleotide fluorescence from the cornea. Invest Ophthalmol Vis Sci 1980; 19: 96-102.

4 Tsubota K, Krauss JM, Kenyon KR, Laing RA, Miglior S, Cheng H-M. Lens redox fluorometry: pyridine nucleotide fluorescence and analysis of diabetic lens. Exp Eye Res 1989; 49: 321-4.

5 Tsubota K, Laing RA, Chiba K, Hanninen L, Kenyon KR. Noninvasive metabolic analysis of preserved rabbit coninvasive metabolic analysis of prese

6 Shimazaki J, Tornheim K, Laing RA. Correlation of redox fluorometry and analytical measurements of pyridin nucleotide. Invest Ophthalmol Vis Sci 1989; 30: 2274-8.

7 Shimazaki J, Tsubota K, Hayashi K, Kenyon KR, Lain $R A$. Distribution of autofluorescence in the rabbit cornea epithelium. Ophthalmic Res 1993; 25: 220-5.

8 Shimazaki J, Tsubota K, Yoshida A, Tornheim K, Laing RA. Changes of corneal redox state in diabetic animal models. Cormea 1995; 14: 196-201.

9 Chance B, Schoener B, Oshino R, Itshak F, Nakase Y. Oxidation-reduction ratio studies of mitochondria in freeze-trapped samples. $\mathcal{f}$ Biol Chem 1979; 254: 4764-71.

10 Masters BR. Noninvasive corneal redox fluorometry. In Zadunaisky JA, Davson $\mathrm{H}$, eds. Current topics in eye Zadunaisky JA, Davson H, eds. Current topics in
research. Orlando: Academic Press, 1984: 139-200.

11 Ota S, Laing RA, Oak SS, Yang ZR. Metabolic changes a the wound margin of the rabbit corneal endothelium Invest Ophthalmol Vis Sci (Suppl) 1990; 31: 319.

12 Tuberville AW, Wood TO, McLaughlin BJ. Cytochrome oxidase activity of Fuchs' endothelial dystrophy. Curr Eye Res 1986; 5: 939-47. 